\title{
Multiple Wavelength Geodesy
}

Judah Levine *

Time and Frequency Division, National Bureau of Standards Boulder, Colorado 80302

Abstract. We are constructing an apparatus which should be able to measure baselines up to $50 \mathrm{~km}$ long with a fractional uncertainty of about $5 \times 10^{-8}$. The instrument will measure both the optical length and the required correction due to the refractivity of the atmosphere using three wavelengths transmitted in one direction over the path to an active receiver. The three wavelengths are $632.8 \mathrm{~nm}, 441.6 \mathrm{~nm}$ and $3.7 \mathrm{~cm}$. The two endpoint instruments are synchronized using subsidiary return transmissions at 632.8 $\mathrm{nm}$ and another telemetry signal. The one-way nature of the system allows an increase in range over existing round-trip systems.

Geodetic measurements have important bearing on many areas of tectonophysics such as plate tectonics and earthquake processes. In particular precise geodetic measurements are needed to understand how strain fields near plate boundaries change with time and why there are stresses in the interiors of plates large enough to cause isolated zones of seismicity.

These questions are being addressed using fixed geophysical instruments and portable instruments. Although one instrument might be used for all geodetic measurements, there may be some advantage to considering different techniques for the portable and fixed measurement programs.

In the case of a permanent observatory, weight and size are second order considerations, and emphasis should be placed on accuracy and sensitivity.

Berger and Levine (1974) have estimated the power spectrum of the random "fluctuations in strain over a wide frequency range. They concluded that the power spectrum is inversely proportional to the square of the frequency, and that the power at $1 \mathrm{~Hz}$ is approximately $8 \times 10^{-28}$ $(\Delta \mathrm{L} / \mathrm{L})^{2} / \mathrm{Hz}$.

The important point is that they used two very different instruments located $2000 \mathrm{~km}$ apart in very different geologies. The two spectra are essentially identical. In spite of these differences. This suggests that both instruments are limited by the same processes within the earth and that their power spectra represent a lower bound to what might be obtained using other techniques.

Their results do not support the widely-held beliefs that installations in mines or tunnels present insuperable problems and that long ( 800 meter) baselines are $a$ priopi better than shorter (30 meter) ones. The instruments appear to be limited by pier tilt, local effects, and, pos-

\footnotetext{
*Fellow, Joint Institute for Laboratory Astrophysics of the National Bureau of Standards and the University of Colorado.
}

Proc. of the 9h GEOP Conference, An Imernational Symposium on the Applicutions of Geodesy to Geodymumics, October 2-5, 1978, Dept. of Geodetic Science Rept. No. 280. The Ohio State Univ.. Columbus, Ohio 432 to. sibly, by drift in the wavelength stabilizer. These are correctable, at least in principle, so that it is not unreasonable to expect that a carefully designed instrument of moderate length (of order 100 meters) could be installed a meter or two below ground level, and that such an instrument would be limited by earth noise for periods shorter than a few years. Furthermore, readout schemes are possible which would obviate the need to operate the instrument continuously.

A strainmeter of this type would have only two weaknesses. First, it would be impractical to move to a new site since a significant fraction of the cost of the system comes from site preparation and installation. Second, it might be influenced by local effects such as tilting of the piers or shears within the ground in the immediate vicinity of the piers.

These local effects were not a problem in the Poorman Mine because it was a hard-rock site far below ground level, but it is possible that they will be the dominant problem at surface (or near surface) sites even if the material appears to be competent.

Berger has studied these problems extensively at the Piñon Flat Observatory. He finds significant correlations between rainfall and changes in strain rate and an anomalous shear in the top few meters of the ground under the pier (Berger, 1978). He concludes that the only way to deal with this problem is to refererice'the endpoints to deeper, presumably more stable, rock.

These sorts of difficulties will limit measurements made with instruments of any length. Anomalous pier motions on the order of millimeters represent fractional changes of parts in $10^{8}$ even over $50 \mathrm{~km}$ baselines, so that such effects will make significant contributions to the error budget of any instrument now in operation or under construction. It is very important that these effects be studied in detail. It would be especially useful to compare measurements made In the same area by instruments using very different length baselines.

Laser strainmeters are clearly unsultable if the instrument must be portable. In this case we must use an instrument capable of measuring distances through the atmosphere. There are many Instruments which have been designed to perform such measurements (Slater and Huggett, 1976). Since all such instruments effectively measure the transit time for some electromagnetic signal, it is necessary to know the actual speed of light in the atmosphere in order to derive the distance.

The refractivity (the deviation of the index of refraction from unity) of the atmosphere is about $3 \times 10^{-4}$ for visible wavelengths. Since it is desirable to measure distances with a fractional uncertainty of less than $1 \times 10^{-7}$, the atmospheric correction is very important. The refractivity is a function of atmospheric tem- 


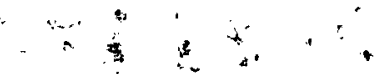

perature, pressure and humidity so that making the corrections using meteorological data is cumbersome but possible. For optimum results the atmosphere must be sampled along the path, usually using sensors carried by an airplane (Savage and Prescott, 1973).

Methods which determine the refractivity of the atmosphere by measuring its dispersion (i.e., the apparent difference in distance between measurements made using different wavelengths) have been proposed for some years (Bender and Owens, 1965).

The use of two optical wavelengths, for example, enables one to determine the dry-air contribution to the refractivity, but does not correct for the refractivity due to water vapor, since the refractivity of water vapor is almost non-dispersive across the visible spectrum. The addition of a third measurement at a microwave frequency allows nearly perfect determination of the index except for a small term which can almost always be determined using temperature measurements at the endpoints. An analysis by Thayer (1967) of such a three-wavelength system suggests that baselines up to $50 \mathrm{~km}$ long could be measured with fractional uncertainties of $3 \times 10^{-8}$, and that the main 1imitation on the accuracy of such a measurement would come from the difference in the paths traversed by the two optical wavelengths due to the vertical gradient in the refractivity of the atmosphere.

Instruments which measure the refractivity of the atmosphere using multiple wavelength methods have been described by Slater and Huggett (1976) and by others (Wood, 1971). The one shortcoming of these instruments is that they can measure only rather short baselines (up to $15 \mathrm{~km}$ ). These instruments are limited by spreading and attenuation of the beam in the atmosphere and by an Inability to totally distinguish between a true retum signal and 11 ght scattered backwards from the exit optics of the transmitter.

If these systems are converted to one-way operation by replacing the retroreflector by an active receiver and a second transmitter, then a large Increase in retumed signal will result. The increase in range realized by conversion to one-way operation is at least a factor of two (if the signal-to-noise ratio is 1imited primarily by attenuation) and may be considerably more than that.

The simplified schematic diagram of such an instrument is shown in Figure 1. Light from the source is sent through the local modulator, traverses the path, and then goes through the farend modulator before detection. After being twice modulated the light at the detector will show variation at the difference frequency $f_{b}=f_{1}-f_{2}$ (the other, higher, frequency components are not detectable). If two different optical wavelengths are sent through the system simultaneously, the two signals at the far end will show a phase difference proportional to the refractivity of the atmosphere. If a third 1ight source sends light backwards through the system, It will arrive at the original end with a phase proportional to the transit time along the path and the various oscillator offsets. If a secondary 1ink is used to transmit synchroni:ing information between the two ends then both the dispersion and the distance may be determined. This link is most conventently done using a microwave carrier, in which case the phase shift of the microwave carrier provides information

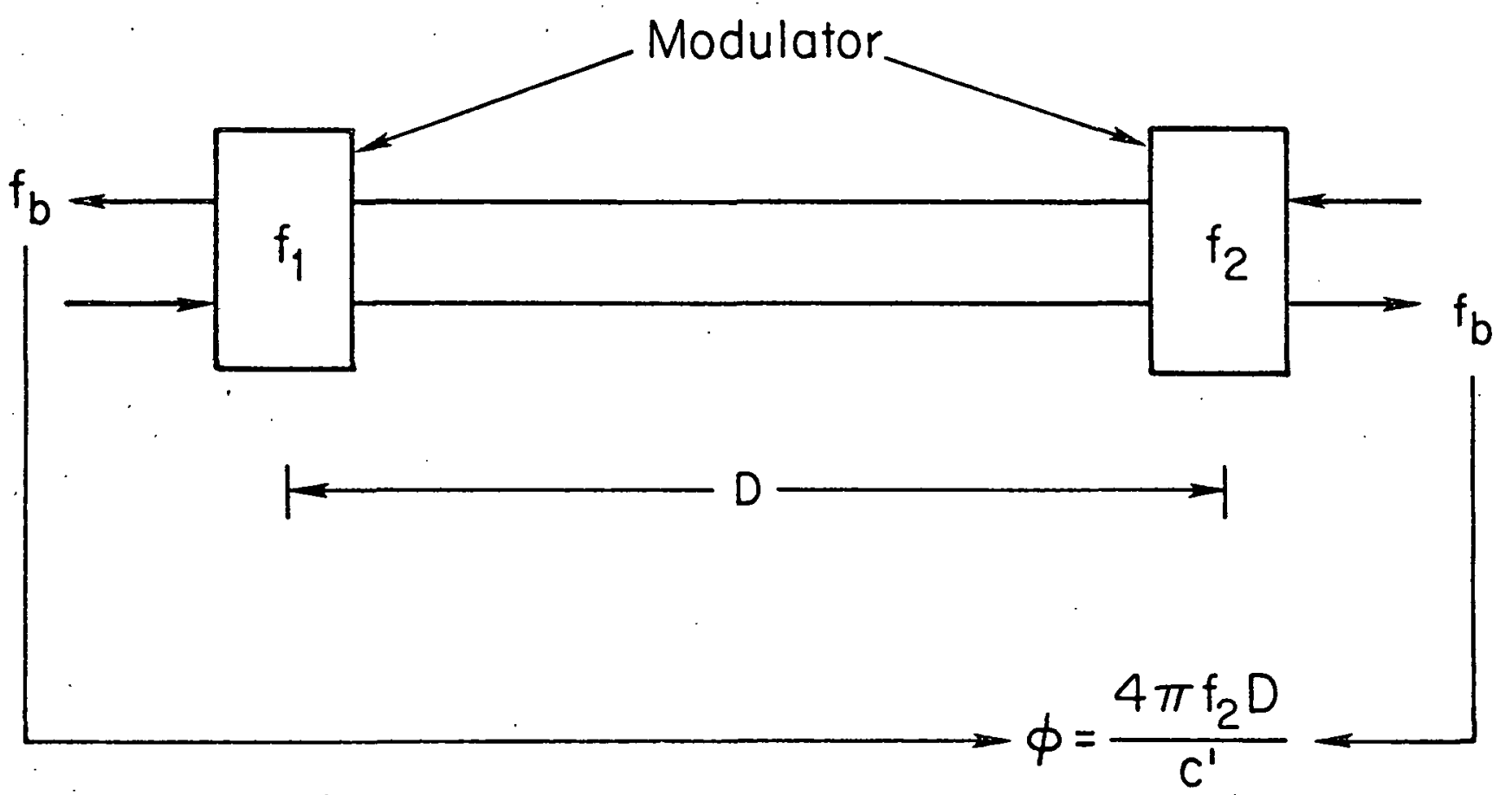

Fig. 1. Principle of two-way optical distance measurement. Note that the measured phase depends only on the frequency of the oscillator at the end where the measurements are made. 
about the contribution of atmospheric water vapor to the refractivity.

Figure 2 shows a block diagram of the complete instrument. The dispersion measurements are made using $441.6 \mathrm{~nm}, 632.8 \mathrm{~nm}$ and $8.1 \mathrm{GHz}$. The red $(632.8 \mathrm{~nm})$ signal is sent back along the path for the distance measurement.

Figure 3 shows the calculated range of the instrument, and compares it with a retroreflecting instrument using similar technology. The lower three curves are for the retroreflecting instrument and the upper ones for the two-way design. Both systems are assumed to use $5 \mathrm{~mW}$ lasers with $1 \%$ overall detection efficiency. The range is defined to be the distance at which shot noise limitations allow a measurement with an uncertainty at $1 \times 10^{-8}$ in 10 seconds. We feel that an angular beam spread of 10 arcseconds is probably realistic for a typical atmosphere.

An instrument designed according to these principles is currently under construction. This instrument can be easily broken down into pleces, the heaviest of which weighs about $60 \mathrm{~kg}$ so that it can be assembled by two people at any location accessible by a four-wheel drive vehicle or a helicopter.
It is possible to use the principles of the multiple wavelength system to construct a three wavelength refractometer. Such an instrument would measure the index of refraction of the path but would not measure the distance.

Although such an instrument would use essentially the same hardware as the distance-measuring instrument, the electronics are measurably simpler and no return transmissions are necessary.

Such an instrument might prove useful as an adjunct to existing geodetic instruments.

This work is supported in part by NASA Grant No. NSG-7344 through the University of Colorado.

\section{References}

Bender, P.L. and J.C.. Owens, Correction of optical distance measurements for the fluctuating atmospheric index of refraction, J. Geophys. Res., 70, 2461-2, 1965.

Berger, J., private communication, 1978.

Berger, J. and J. Levine, The spectrum of earth strain from $10^{-8}$ to $10^{2} \mathrm{~Hz}, \mathrm{~J}$. Geophys. Res., 79, 1210-4, 1974 .

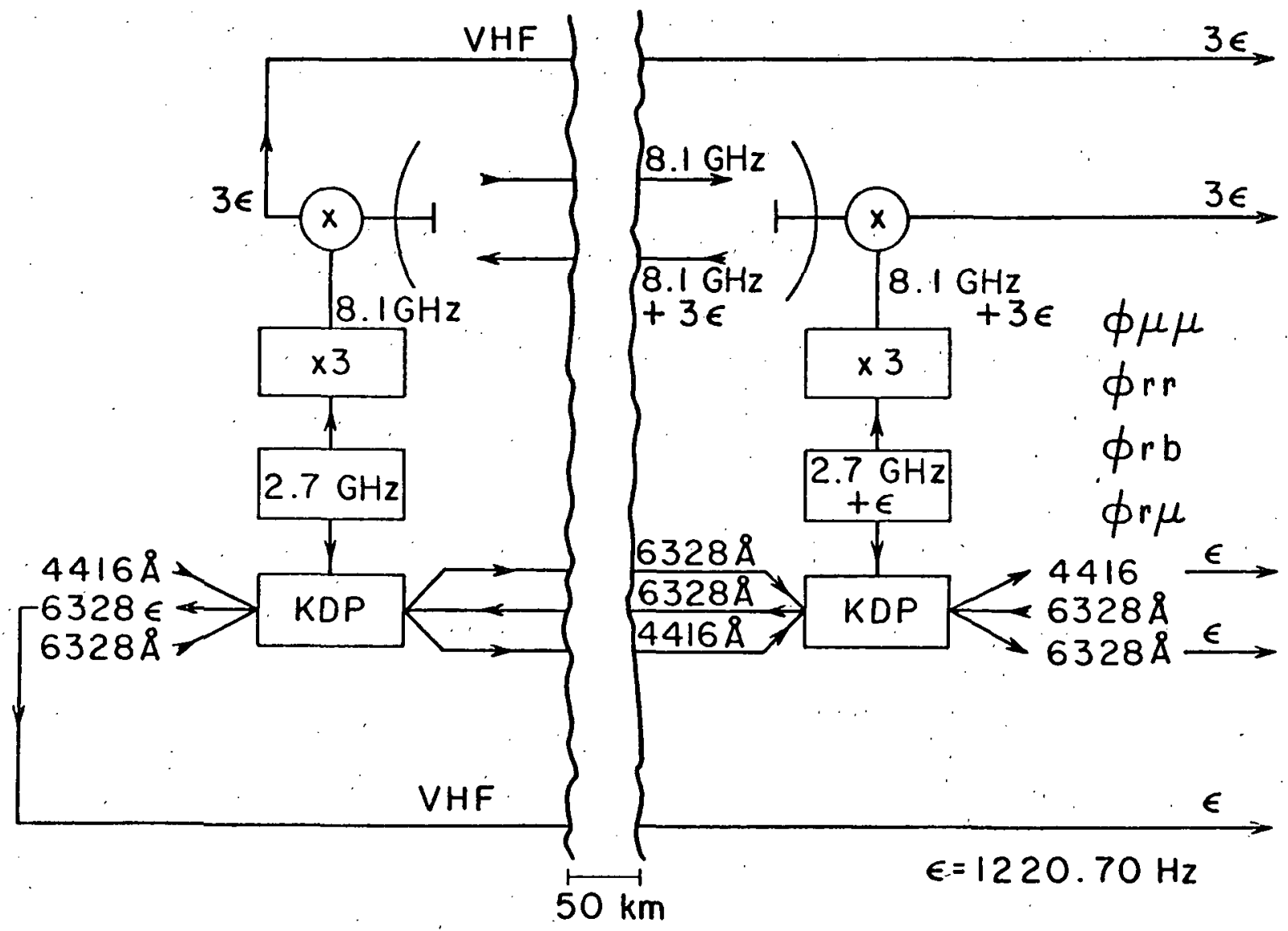

Fig. 2. Block diagram of the prototype system.

"UHF" is a low bandwidth telemetry channel. The various phase measurements are identified with subscripts $\mathrm{r}$ for red $(632.8 \mathrm{~nm}), \mathrm{b}$ for blue $(441.6 \mathrm{~nm})$ and $\mu$ for microwave $(8.1 \mathrm{GHz})$. 


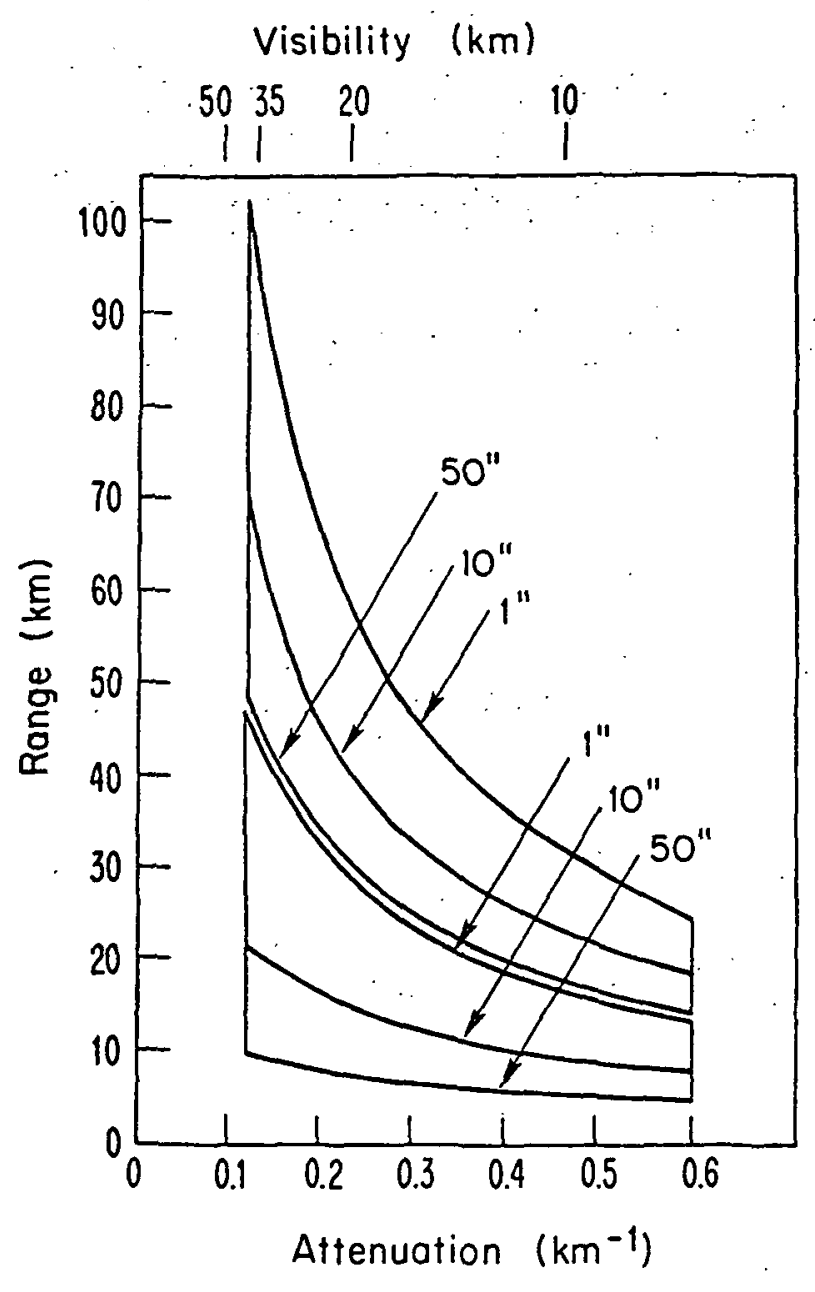

Fig. 3. Calculated range of one-way (upper curves) and retroreflector EDM instruments as a function of optical beam spread and atmospheric attenuation.
Savage, J.C. and W.H. Prescott, Precision of geodolite distance measurements for determining fault movements, J. Geophys. Res., 78, 6001-8, 1973.

Slater, C.R. and G.R. Huggett, A multiwavelength distance measuring instrument for geophysical experiments, J. Geophys. Res., 81, 6299-6305, 1976.

Thayer, G.C., Atmospheric effects of multiple frequency range measurements, Technical Report IER 56-ITSA 53, Environmental Science Services Administration, Rockville, MD, 1967.

Wood, L.E., Progress in electronic surveying, U. S. National Report, EOS, 52, IUGG17-21, 1971. 